\title{
EFEITOS DE UM PROGRAMA DE GINÁSTICA LABORAL SOBRE SINTOMAS DE DOR E FADICA EM TRABALHADORES
}

\author{
LABOR GYMNASTIC'S EFFECTS ON PAIN AND FATIGUE \\ SYMPTOMS IN WORKERS
}

\section{Carolina Franciscatto', Juliana Costa da Rocha', Simone Lara²}

\section{RESUMO}

Objetivo: Avaliar os efeitos de um programa de ginástica laboral sobre os sintomas de dor e fadiga em servidores públicos municipais. Metodologia: 0 estudo ocorreu durante 08 semanas, onde participaram 23 trabalhadores, divididos em Grupo 1 (G1), que receberam palestras gerais em saúde e Grupo 2 (G2), que participaram do programa de Ginástica Laboral. Foram avaliados, antes e após intervenção, os sintomas de fadiga (questionário de Yoshitake), e os sintomas de dor cervical e lombar (Índice de Incapacidade do Pescoço ou IPP e Roland Morris, respectivamente). Foram utilizadas análises descritivas, teste $t$ de student pareado e teste Wilcoxon. Resultados: Houve redução de sintomas associados às dores cervicais e à fadiga no G2, o que não ocorreu no G1. Conclusão: 0 estudo evidenciou um impacto positivo de um programa de ginástica laboral na redução de dor e sintomas de fadiga em uma amostra de trabalhadores.

Descritores: Saúde do Trabalhador; Fadiga; Cervicalgia; Dor Lombar.

\section{ABSTRACT}

Objective: To investigate the effects of a Labor Gymnastic's program on pain and fatigue symptoms in municipal public workers. Methodology: Study occurred during 08 weeks, and 23 participants were included, divided into group 1 (G1), which received health general lectures and group 2 (G2), which participated the Labor Gymnastic's program. Fatigue symptoms (Yoshitake questionnaire), and cervical and lumbar pain symptoms (Neck Disability Index and Roland Morris questionnaire, respectively) were assessed before and after intervention. Descriptive analyses, paired t-test and Wilcoxon test were performed. Results: There was a reduction of cervical pain symptoms and fatigue symptoms only in G1. Conclusion: The study showed a positive impact of a Labor Gymnastic's program in reducing pain and fatigue symptoms in this sample.

Descriptors: Occupational Health; Fatigue; Neck Pain; Low back Pain.
${ }^{1}$ Graduada em Fisioterapia pela Universidade Federal do Pampa (UNIPAMPA), Uruguaina, RS, Brasil.

2 Doutora em Educação em Ciências: Química da vida e saúde pela Universidade Federal de Santa Maria (UFSM), Santa Maria, RS, Brasil. 


\section{Introdução}

As afecções osteomusculares vêm aumentando nos trabalhadores dos vários setores da economia no Brasil, sendo consideradas como um preocupante problema de saúde devido as repercussões causadas à sociedade ${ }^{1}$. Nesse contexto, a exposição às diversas condições de trabalho pode gerar demandas no trabalhador, resultando no surgimento de doenças relacionadas ao trabalho².

Os distúrbios do sistema musculoesquelético no trabalho ocorrem, frequentemente, quando a demanda física do trabalho excede a capacidade física do trabalhador ${ }^{3}$. A adoção de posturas ocupacionais inadequadas, esforço repetitivo, trabalhos com cargas e períodos elevados, ausência de intervalos e inadequação ergonômica são fatores que contribuem para o desenvolvimento das doenças ocupacionais ${ }^{4}$. Adicionalmente, uma experiência de cansaço, desgosto pela atividade atual e falta de vontade para continuar resultam na situação de fadiga do trabalhador ${ }^{5}$.

Considerando que as disfunções ocupacionais possuem, como características comuns, a dor crônica e o desgaste de estruturas do sistema musculoesquelético ${ }^{6}$, torna-se relevante a construção de estratégias que tenham como enfoque a promoção da saúde do trabalhador. Sob essa perspectiva, promover o bem estar dos trabalhadores é de suma importância para assegurar uma maior produtividade e qualidade no trabalho e maior satisfação na vida familiar e pessoal ${ }^{7}$.

Sob esse olhar, uma das estratégias para essa finalidade é o desenvolvimento de programas de ginástica laboral, que representam uma atividade física realizada durante a jornada de trabalho, incluindo exercícios de compensação aos movimentos repetitivos ou a posturas desconfortáveis assumidas durante o período de trabalho ${ }^{8}$. A ginástica laboral visa a prevenção e a promoção da saúde do trabalhador através de reeducação das posturas solicitadas no ambiente de trabalho, por prevenir as doenças ocupacionais e por minimizar a fadiga ocasionada pela execução das atividades de trabalho ${ }^{9}$.

Com base no exposto, o objetivo desse estudo foi avaliar a influência de um programa de ginástica laboral sobre os sintomas de dor e de fadiga em uma amostra de servidores públicos municipais.

\section{Metodologia}

Trata-se de um estudo de intervenção e quantitativo, no qual foram convidados a participar 40 servidores públicos municipais, em atividades laborais alocados na Secretaria Municipal de Indústria, Comércio, Turismo e Trabalho, na Prefeitura Municipal de Uruguaiana. O estudo ocorreu no período de abril à junho de 2015. Os critérios de inclusão do estudo foram: aceitar voluntariamente participar do trabalho e ter frequência mínima de $75 \%$ de participação do projeto. Os critérios de exclusão foram: gestantes e profissionais com previsão de afastamento durante o período do estudo.

Após a análise desses critérios, 10 trabalhadores não integraram a proposta devido à afastamento do trabalho no momento do estudo e 07 servidores decidiram não participar. Assim, participaram efetivamente do estudo 23 servidores, divididos aleatoriamente em dois grupos, constituindo o Grupo 1 (G1) e o Grupo 2 (G2). 0 G1 (N=12) recebeu apenas palestras gerais em saúde e o $\mathrm{G} 2(\mathrm{~N}=11)$ participou do programa de ginástica laboral. $\mathrm{O}$ estudo foi aprovado pelo Comitê de ética e pesquisa da Universidade Federal do Pampa (número 939.616 em 27/01/2015), e os interessados em participar, após explanação prévia do projeto pelos pesquisadores, assinaram o Termo de Consentimento Livre e Esclarecido (TCLE).

O G1 e o G2 foram avaliados e reavaliados por meio de um protocolo, incluindo:

- Questionário sobre os dados pessoais - os trabalhadores responderam dados sobre o sexo e a idade;

- Questionário de Fadiga proposto por Yoshitake - Instrumento que investiga a presença de fadiga, composto por questões fechadas e semi-abertas, sendo de fácil aplicação ${ }^{10}$. Apresenta três subgrupos de sintomas de malestar, relacionados a sentimentos gerais de incongruidade, cansaço mental e sensações específicas de cansaço ${ }^{11}$. 0 questionário consta de 10 questões em cada domínio, totalizando 30 questões, no qual o maior escore obtido a partir dessa ferramenta é 30, caracterizando grau máximo de fadiga/mal estar ${ }^{11}$;

- Índice de Incapacidade do Pescoço (IPP) - Instrumento que avalia a incapacidade e a dor na coluna cervical ${ }^{12}$. É formado por dez itens, e cada um é composto por seis alternativas de resposta que se referem a uma atividade de vida diária, com exceção da questão cinco, sobre cefaléia. As alternativas, numeradas de zero à cinco, descrevem graus crescentes de interferência da dor cervical sobre a realização da atividade questionada. $O$ cálculo dos escores é obtido pela soma dos pontos e subsequente conversão do resultado em um valor percentual, sendo considerados apenas os itens respondidos pelo indivíduo ${ }^{13}$; 
- Questionário Roland Morris - Instrumento que avalia a interferência da lombalgia na função do indivíduo, e consiste de 24 itens que descrevem situações de vida diária que podem ser influenciadas por dores lombares. Escores mais altos indicam maior incapacidade ${ }^{14}$.

Após a avaliação, o G2 participou do protocolo de ginástica laboral, durante 08 semanas, sendo 24 encontros, com frequência de três vezes por semana e duração de 50 minutos cada encontro. Cabe ressaltar que o setor disponibilizou o período de 50 minutos para a realização dos encontros com os trabalhadores, uma vez que frequentemente tais encontros dos programas de ginástica laboral ocorrem em um período menor de tempo.

0 protocolo de ginástica laboral consistiu em alongamentos corporais ${ }^{15}$ (quadro 1), e exercícios ativos para membros superiores e inferiores, coluna cervical e lombar, cintura escapular e pélvica ${ }^{16}$ (quadro 2).

Quadro 1 - Protocolo de alongamentos

\begin{tabular}{|c|c|}
\hline Objetivo & Descrição \\
\hline Alongar musculatura cervical & $\begin{array}{l}\text { Inclinação lateral da cabeça, mantendo } \\
\text { por } 15 \text { segundos (para cada lado). }\end{array}$ \\
\hline $\begin{array}{c}\text { Alongar musculatura clavicular e } \\
\text { deltoides }\end{array}$ & $\begin{array}{l}\text { Extensão de ombro, flexão de punho } \\
\text { (mãos entrelaçadas na altura de coluna } \\
\text { lombar), mantendo } 15 \text { segundos. }\end{array}$ \\
\hline Alongar musculatura coluna lombar & $\begin{array}{l}\text { Flexão de tronco, ombros em flexão, } \\
\text { encostar mãos próximas aos pés, } \\
\text { mantendo } 15 \text { segundos. }\end{array}$ \\
\hline Alongar flexores laterais de tronco & $\begin{array}{c}\text { Em semiflexão de joelhos, membros } \\
\text { superiores em flexão de ombros (mãos } \\
\text { entrelaçadas em cima da cabeça, } \\
\text { realizar flexão lateral de tronco, } \\
\text { mantendo a posição por } 15 \text { segundos } \\
\text { (para cada lado). }\end{array}$ \\
\hline Alongar musculatura de peitorais & $\begin{array}{l}\text { Em pé, em extensão de ombros, } \\
\text { com mãos entrelaçadas na altura de } \\
\text { coluna lombar, manter a postura por } 15 \\
\text { segundos. }\end{array}$ \\
\hline Alongar flexores de quadril & $\begin{array}{l}\text { Realizar flexão máxima de joelhos, } \\
\text { mantendo por } 15 \text { segundos cada perna. }\end{array}$ \\
\hline Alongar isquiotibiais e tríceps sural & $\begin{array}{l}\text { Em pé, realizar flexão máxima de } \\
\text { tronco, mantendo por } 15 \text { segundos. }\end{array}$ \\
\hline
\end{tabular}

Fonte: Clemenceau, 2012.

Quadro 2 - Protocolo de exercícios

\begin{tabular}{|c|c|}
\hline Exercício/Objetivo & Descrição \\
\hline Exercícios de mobilidade da coluna \\
cervical & Em pé, com os membros superiores \\
& dispostos ao longo do corpo, realiza \\
& flexão e extensão de coluna cervical \\
& (3 séries de 8 repetições). Após esse \\
& exercício, seguindo mesma posição \\
& inicial, realizar inclinação lateral de \\
& coluna cervical (3 séries de 5 repetições \\
& para cada lado). \\
\hline
\end{tabular}

Continua... 


\begin{tabular}{|c|c|}
\hline Exercício/Objetivo & Descrição \\
\hline Exercício para flexores de cotovelo & $\begin{array}{l}\text { Em pé, com antebraço em supinação, } \\
\text { realizar flexão/extensão de cotovelos (3 } \\
\text { séries de } 8 \text { repetições). }\end{array}$ \\
\hline Exercício de prono-supinação & $\begin{array}{l}\text { Em pé, com os } 90^{\circ} \text { de abdução } \\
\text { de ombro, realizar movimento de } \\
\text { supinação de antebraço, seguido de } \\
\text { pronação (3 séries de } 8 \text { repetições). }\end{array}$ \\
\hline $\begin{array}{c}\text { Exercício de mobilidade para } \\
\text { cintura escapular, coluna vertebral e } \\
\text { membros superiores }\end{array}$ & $\begin{array}{c}\text { Em pé, com os membros superiores } \\
\text { dispostos ao longo do corpo, realizar } \\
\text { circundução de coluna cervical, } \\
\text { alternada por abdução de ombro em } \\
180^{\circ} \text { (3 séries de } 5 \text { repetições para cada } \\
\text { movimento). }\end{array}$ \\
\hline $\begin{array}{l}\text { Exercício para mobilidade de tronco } \\
\text { (rotação) }\end{array}$ & $\begin{array}{c}\text { Em pé, com tronco e cabeça alinhados, } \\
\text { com as mãos na cintura, realizar rotação } \\
\text { de tronco ( } 3 \text { séries de } 5 \text { repetições para } \\
\text { cada lado). }\end{array}$ \\
\hline Exercício de mobilidade escapular & $\begin{array}{l}\text { Em pé, com ombro em } 90^{\circ} \text { de abdução } \\
\text { e cotovelos fletidos, realizar retração } \\
\text { escapular (3 séries de } 8 \text { repetições). }\end{array}$ \\
\hline $\begin{array}{l}\text { Exercício de mobilidade de tronco } \\
\text { (lateral) }\end{array}$ & $\begin{array}{l}\text { Em pé, com os membros superiores } \\
\text { ao longo do corpo, realizar inclinação } \\
\text { lateral, somado de abdução à } 180^{\circ} \text { de } \\
\text { membro superior contralateral ( } 3 \text { séries } \\
\text { de } 5 \text { repetições para cada lado). }\end{array}$ \\
\hline Exercício de mobilidade de punho & $\begin{array}{l}\text { Em pé, com cotovelos fletidos à } 90^{\circ}, \\
\text { realizar flexão e extensão de punho ( } 3 \\
\text { séries de } 8 \text { repetições). }\end{array}$ \\
\hline Exercício de agachamento & $\begin{array}{c}\text { Em pé, com tronco alinhado e mãos na } \\
\text { cintura, realizar flexão e quadril e joelho, } \\
\text { mantendo pés fixos ao solo ( } 3 \text { séries de } \\
8 \text { repetições). }\end{array}$ \\
\hline Exercício de planti/dorsiflexão & $\begin{array}{c}\text { Sentado, com joelhos estendidos, } \\
\text { realizar movimento de plantiflexão, } \\
\text { seguido de dorsiflexão ( } 3 \text { séries de } 8 \\
\text { repetições). }\end{array}$ \\
\hline
\end{tabular}

Fonte: Polito e Bergamaschi, 2002.

O G1 participou de um programa de educação em saúde por meio de temas relacionados à promoção da saúde no ambiente laboral, com duração aproximada de 15 minutos, descrito no quadro 3. 
Quadro 3 - Temas de educação em saúde

\begin{tabular}{|c|c|}
\hline Periodo & Tema \\
\hline $2^{\text {a }}$ semana & $\begin{array}{c}\text { Relevância da aquisição de hábitos } \\
\text { saudáveis para a saúde do trabalhador }\end{array}$ \\
\hline $4^{\text {a }}$ semana & $\begin{array}{c}\text { Importância da atividade física para a } \\
\text { saúde do trabalhador }\end{array}$ \\
\hline $6^{\text {a }}$ semana & $\begin{array}{c}\text { Prevenção de doenças relacionadas ao } \\
\text { trabalho }\end{array}$ \\
\hline $8^{\text {a }}$ semana & $\begin{array}{c}\text { Adequação de mobiliário na prevenção } \\
\text { das DORT }\end{array}$ \\
\hline
\end{tabular}

Fonte: os autores

Para análise dos dados foi utilizado o programa estatístico Graph Pad INSTAT. Após verificação da normalidade dos dados, foi utilizado o teste $t$ de student pareado para as variáveis normais e o teste Wilcoxon para as variáveis com distribuição não normal. Assim, para a comparação pré e pós-intervenção, utilizou-se o teste t de student pareado para as variáveis: sintomas de fadiga e dores cervicais do $\mathrm{G} 2$, e, foi utilizado o teste Wilcoxon para a comparação das variáveis: sintomas de dores lombares no G1 e G2 e sintomas de fadiga e dores cervicais para o G1. Os dados foram apresentados com média e desvio padrão. Foi considerado como significante um $p<0,05$.

\section{Resultados}

Participaram do estudo 12 servidores do G1 (5 do sexo masculino e 7 do sexo feminino, com média de idade de 33,5 anos), e 11 servidores do G2 (6 do sexo masculino e 5 do sexo feminino, com média de idade de 45,5 anos).

A tabela 1 evidencia os sintomas cervicais e lombares do $\mathrm{G} 1$ e do $\mathrm{G} 2$, antes e após o estudo. É possível verificar que não houve modificação dos valores no $\mathrm{G} 1$, porém, houve uma redução significativa nos sintomas cervicais no $\mathrm{G} 2$ após a intervenção.

Tabela 1 - Avaliação dos sintomas de dor cervical e lombar no G1 e no G2, antes e após a intervenção

\begin{tabular}{c|c|c|c|c|c|c}
\hline & \multicolumn{3}{|c|}{ Grupo 1 } & \multicolumn{3}{c}{ Grupo 2 } \\
\cline { 2 - 7 } & Pré & Pós & P & Pré & Pós & P \\
\hline $\begin{array}{c}\text { Sintomas cervicais } \\
\text { (Índice de } \\
\begin{array}{c}\text { incapacidade do } \\
\text { pescoço) }\end{array}\end{array}$ & $5,50 \pm 7,20$ & $5,50 \pm 7,20$ & $\mathrm{P}=0,50$ & $4,72 \pm 5,04$ & $1,81 \pm 2,48$ & $0,005^{*}$ \\
\hline $\begin{array}{c}\text { Sintomas lombares } \\
\text { (Questionário } \\
\text { Roland Morris) }\end{array}$ & $1,41 \pm 3,36$ & $1,41 \pm 3,36$ & $\mathrm{P}=0,50$ & $0,81 \pm 1,25$ & $0,18 \pm 0,40$ & 0,1 \\
\hline
\end{tabular}

*indicam $\mathrm{P}<0,05$

Com relação aos sintomas de fadiga dos trabalhadores do G1, não houve alteração de valores antes e após o estudo. No entanto, foi encontrada uma redução significativa dos sintomas de fadiga no G2 após a intervenção, tanto na pontuação geral dos sintomas, quanto nos três domínios do teste, evidenciando um impacto positivo da ginástica laboral na redução dos sintomas gerais e específicos de mal estar, bem como sobre o cansaço mental (tabela 2). 
Tabela 2 - Avaliação dos sintomas de fadiga no grupo 1 e no grupo 2, antes e após a intervenção

\begin{tabular}{c|c|c|c|c|c|c}
\hline & \multicolumn{3}{|c|}{ Grupo 1 } & \multicolumn{3}{c}{ Grupo 2 } \\
\cline { 2 - 7 } & Pré & Pós & P & Pré & Pós & P \\
\hline Pontuação geral & 10,83 & 10,83 & $\mathrm{P}=0,50$ & 5,90 & 1,81 & $\mathrm{P}=0,0004^{*}$ \\
\hline $\begin{array}{c}\text { Sintomas gerais de } \\
\text { mal estar }\end{array}$ & 4,33 & 4,33 & $\mathrm{P}=0,50$ & 2,18 & 0,45 & $\mathrm{P}=0,0008^{*}$ \\
\hline Cansaço mental & 3,00 & 3,00 & $\mathrm{P}=0,50$ & 2,27 & 0,72 & $\mathrm{P}=0.0076^{*}$ \\
\hline $\begin{array}{c}\text { Sintomas } \\
\text { específicos de mal } \\
\text { estar }\end{array}$ & 3,33 & 3,33 & $\mathrm{P}=0,50$ & 1,45 & 0,63 & $\mathrm{P}=0,02^{*}$ \\
\hline
\end{tabular}

*indicam $\mathrm{P}<0,05$

\section{Discussão}

O presente estudo apresentou resultados significativos quanto à redução dos sintomas de fadiga e de dores cervicais nos servidores que participaram o protocolo de ginástica laboral. Já os integrantes do G1 não obtiveram alteração dos sintomas antes e após o acompanhamento do estudo.

Um estudo atual analisou 25 servidores públicos de uma Instituição Pública de Ensino Superior em Saúde no interior de São Paulo ${ }^{17}$, onde foram avaliadas queixas osteomusculares e nível de fadiga, por meio de instrumentos específicos. 0 protocolo de intervenção teve uma frequência de duas vezes por semana, duração de 30 minutos cada, e ocorreu durante um período de 10 semanas, tendo como base alongamentos do método Isostretching. Após a intervenção, os autores encontraram uma redução significativa dos sintomas de dor osteomusculares e queixas de fadiga nos trabalhadores. Esses dados estão de acordo com os do presente estudo, apresentando um período de aplicação do protocolo semelhante, demonstrando que, embora as intervenções ocorram durante um curto período de tempo, é possível verificar melhoras significativas sobre a dor e fadiga em trabalhadores.

Candotii, Stroschein e Noll ${ }^{18}$ avaliaram os sintomas de dor em 30 trabalhadores do setor administrativo, divididos em grupo controle (GC) e grupo intervenção (GI), onde o $\mathrm{GI}$ praticou um programa de ginástica laboral. Os resultados demonstraram que houve redução significativa dos sintomas relativos à frequência e à intensidade da dor, e a interferência da dor nas atividades diárias somente no Gl, o que não ocorreu no GC. Assim, esses dados vão ao encontro do presente estudo, visto que houve redução significativa dos sintomas de dor (na coluna cervical) no grupo participante da ginástica laboral.

$O$ trabalho de Ferracini e Valente ${ }^{19}$ avaliou os efeitos de um programa de ginástica laboral em 15 funcionários do setor de faturamento de um hospital público. Este programa foi pautado em exercícios de alongamentos, voltados aos grupos musculares mais requisitados na atividade profissional e identificados pelos questionários. Evidenciou-se, após a intervenção, um aumento do bem estar diário e melhora do relacionamento interpessoal, referido pelos trabalhadores. Esses dados corroboram com os do presente estudo, onde houve redução dos sintomas gerais e específicos relacionados ao mal estar dos praticantes da intervenção.

O nível de estresse foi avaliado em 34 funcionários atuantes no setor de produção de uma empresa, divididos em GC e Gl, antes e após a aplicação de um programa de ginástica laboral ${ }^{20}$. Para tal, observou-se uma redução significativa dos sintomas de estresse dos trabalhadores que participaram do programa de ginástica laboral, o que não ocorreu com o GC, reiterando a importância da prática de atividade física durante a jornada de trabalho de trabalhadores. Nomeadamente, Resende et al. ${ }^{21}$ também encontraram resultados favoráveis da ginástica laboral sobre as variáveis de estresse e disposição para o trabalho em 24 funcionários de um tele-atendimento. Coulson, McKenna e Field ${ }^{22}$ reportam efeitos positivos da ginástica laboral, sobre o desempenho no trabalho, em trabalhadores de empresas privadas e públicas. Embora, no presente estudo, as variáveis de estresse e de disposição e desempenho no trabalho não tenham sido avaliadas, houve redução dos sintomas de mal estar, mensurados através do questionário de Fadiga utilizado no 
presente estudo. Dessa forma, foi possível observar, através de relatos dos trabalhadores, uma redução do nível de estresse e um aumento da disposição dos mesmos nas atividades laborais.

O trabalho apresenta como limitações o número reduzido de participantes, tendo em vista que foram incluídos trabalhadores de apenas uma secretaria, a fim de atender ao horário dos voluntários e assiduidade. Além disso, uma outra limitação importante foi o curto período de tempo da intervenção proposta.

\section{Considerações Finais}

O presente estudo apresentou resultados favoráveis de um programa de ginástica laboral sobre a redução de sintomas associados à dor cervical e à fadiga, em um grupo de servidores públicos municipais. Esse fato evidencia a importância da inserção desses programas, envolvendo a prática de atividade física no ambiente de trabalho, contribuindo para a promoção da saúde do trabalhador e à prevenção de lesões ocupacionais.

\section{Referências}

1. Martins AC. Sintomas osteomusculares relacionados ao trabalho de enfermagem em unidade de terapia intensiva [dissertação]. São Paulo: Escola de Enfermagem: Universidade de São Paulo; 2011.

2. Kowalski C, Driller E, Ernstmann N, Alich S, Karbach U, Ommen O, et al. Associations between emotional exhaustion, social capital, workload, and latitude in decision-making among professionals working with people with disabilities. Res Dev Disabil. 2010;31(2):470-9.

3. Alencar MCB, Schultze VM, Souza SD. Distúrbios osteomusculares e o trabalho dos que cuidam de idosos institucionalizados. Fisioter Mov. 2010;23(1):63-72.

4. Carugno M, Pesatoril AC, Ferrario MM, Ferrari AL, da Silva FJ; Martins AC, et al. Physical and psychosocial risk factors for musculoskeletal disorders in Brazilian and Italian nurses. Cad. Saúde Pública. 2012:28:1632-1642.

5. Kant IJ, Bültmann U, Schröer KAP, Beurskens AJHM, Van Amelsvoort LGPM, Swaen GMH. (2003). An epidemiological approach to study fatigue in the working population: The Maastricht cohort study. Occupational and Environmental Medicine, 60(Suppl 1), 32-39.

6. Neves RF, Nunes MO. Da legitimação a (res)significação: o itinerário terapêutico de trabalhadores com LER/DORT. Ciênc Saúde Coletiva. 2010; 15(1):211-20.

7. Aquino A de S, Fernandes ACP. Qualidade de vida no trabalho. J Health Sci Inst. 2013;31(1):53-8.

8. Andrade AM de, Reuter CP, Reckziegel MB, Pohl HH, Burgos LT. Ginástica Laboral: efeitos de um programa de ginástica laboral sobre a flexibilidade em trabalhadores de diversos setores de um hospital de ensino da região do Vale do Rio Pardo/RS. Cinergis 2015;16(3):209-213.

9. Monteiro JÁ, Lyrio AS, Gomes CS, Rangel GMB, Silva LC. Benefícios da ginástica laboral como medida de prevenção para doenças ocupacionais: um estudo sob a ótica da ergonomia. Persp. Online: biol. \& saúde. 2015;18(5):32-33.

10. Panobianco MS, Magalhães PAP de, Soares CR, Sampaio BAL, Almeida AM de,Gozzo T de O. Prevalência de depressão e fadiga em um grupo de mulheres com câncer de mama. Rev Eletr Enf. 2012; 14(3): 532-540.

11. Yoshitake $H$. There are characteristic patterns of subjective fatigue symptoms. Ergonomics. 1978;21(3): 231-233.

12. Cook C, Richardson JK, Braga L, Menezes A, Soler X, Kume P. et al. Cross-cultural adaptation and validation of the Brazilian Portuguese version of the neck disability index and neck pain and disability scale. Spine. 2006;31(14): 1621-1627. 13. Vernon H, Mior S. The neck disability index: a study of reliability and validity. J Manipulative Physiol Ther. 1991;14(7): 409-405.

14. Nusbaum L, Natour J, Ferraz MB, Goldenberg J. Translation, adaptation and validation of the Roland-Morris questionnaire - Brazil Rolan-Morris. J Med Biol Res. 2001;34(2): 203-210.

15. Clémenceau J. Guia de alongamento: abordagem anatômica ilustrada. Barueri: Manole; 2012.

16. Polito E, Bergamaschi EC. Ginástica laboral: teoria e prática. Rio de Janeiro: Sprint; 2002.

17. Swerts FCT de F. Efeitos do Isostretching na redução de queixas de dor osteomuscular, fadiga e na melhora da flexibilidade em funcionários públicos. Ribeirão Preto: [s.n.], 2014. 222p. Tese (doutorado) - Universidade de São Paulo, 2014.

18. Candotii CT, Stroschein R, Noll M. Efeitos da ginástica laboral na dor nas costas e nos hábitos posturais adotados no ambiente de trabalho. Rev. Bras. Ciênc. Esporte. 2011;33(3): 699-714. 
19. Ferracini NG, Valente FM. Presença de sintomas musculoesqueléticos e efeitos da ginástica laboral em funcionários do setor administrativo de um hospital público. Rev Dor. 2010;11(3): 233-236.

20. Tanaka K, Deutsch S, Valiam P. Efeito da ginástica laboral no estresse. EF Deportes Revista Digital /Buenos Aires. 2007;12(108).

21. Resende MCF, Tedeschi CM, Bethônico FP, Martins TTM. Efeitos da Ginástica Laboral em Funcionários de Teleatendimento. Acta Fisiatr. 2007;14(1): 25 -31.

22. Coulson JC, McKenna JC, Field M. Exercising at work and self-reported work performance. International Journal of Workplace Health Management. 2008;3(1): 176 - 197.

\section{Simone Lara}

Endereço para correspondência - Universidade Federal do Pampa (UNIPAMPA);

Faculdade de Ciências da Saúde, Campus Uruguaiana, BR 472, km 592,

CP 118, CEP 97500-970, Urugaiana, RS, Brasil.

E-mail: simonelara@unipampa.edu.br

Lattes: http://lattes.cnpq.br/5130937270549081

Carolina Franciscatto - carol_polenta@yahoo.com.br

Juliana Costa da Rocha - julianac_r@hotmail.com

Enviado em 18 de julho de 2015. Aceito em 18 de julho de 2016. 